\title{
Spectrum of Cutaneous Manifestations of Diabetes Mellitus: An Observational Study from a Tertiary Care Hospital
}

\author{
Arjun Baidya ${ }^{1^{*}}$, Ashim Kumar Halder ${ }^{2}$ and Pijush Kanti Datta ${ }^{3}$
}

${ }^{1}$ Department of Endocrinology, N.R.S Medical College, Kolkata, West Bengal, India

${ }^{2}$ Consultant Dermatologist, Contai Subdivision Hospital, Contai, West Bengal, India

${ }^{3}$ Department of Dermatology, Medical College Hospital, Kolkata, West Bengal, India

\begin{abstract}
Introduction: Diabetes leads to destruction all over the body and the skin is not exempted from the attack. The skin shares both the effects of acute gross metabolic derangement and chronic degenerative changes of diabetes, as it are an actively metabolizing tissue.
\end{abstract}

Aims and objectives: To study the clinical patterns of dermatological manifestations of diabetes mellitus in the region of eastern India.

Methods: Sixty consecutive subjects with diabetes mellitus having dermatological manifestations and satisfying the inclusion criteria were included in this study.

Results: Bacterial infections were the commonest dermatological disorder (46.7\%). Different forms of dermatophyte infections and candidiasis were found in $30 \%$ and $23.3 \%$ subjects, respectively. Diabetic dermopathy or 'shin spot' and diabetic foot with gangrenous changes were found in $20 \%$ and $13.3 \%$ subjects, respectively. Skin manifestations associated with diabetes were the second most common skin disorders and constituted $43.3 \%$ (26 out of 60 cases). Pruritus of unknown origin and xerosis (13.3\%) were the most common cutaneous abnormalities in the group associated with diabetes. Other cutaneous manifestations, namely pigmented purpuric dermatosis $(6.6 \%)$, psoriasis $(3.3 \%)$, vitiligo $(6.6 \%)$, lichen planus $(3.3 \%)$, porokeartosis $(3.3 \%)$, sclerosis, diabetic thick skin and contracture $(6.6 \%)$, lipodystrophy $(3.3 \%)$, skin rashes $(6.6 \%)$ and scar with abscess $(1.6 \%)$, were very rare.

Conclusions: Our study has provided some insights into the pattern of dermatological manifestations in diabetes mellitus. A larger prospective study will further consolidate our main objective that management of diabetes is a holistic one and due attention on skin complications is warranted.

Keywords: Spectrum; Cutaneous; Manifestations; Diabetes mellitus

\section{Introduction}

Diabetes mellitus (DM) wreaks havoc all over the body, and the skin is not exempted from the attack. Insulin affects the utilization of glucose in skin and is required for growth and differentiation of keratinocytes and fibroblasts.

The cutaneous signs of diabetes are the manifestations of multiple factors. Abnormal carbohydrate metabolism, other altered metabolic pathways, atherosclerosis, microangiopathy, neuronal degeneration and impaired host mechanism all play a role. Although some cutaneous reactions are secondary to treatment, simple skin manifestation may be the first clue to an underlying deadly disease not less than diabetes. This has stimulated interest to all diabetic care providers, inclusive of dermatologists for development of an early detection system for markers of DM, of which skin is a vital organ and which demands due honor in the work up list in connection with diabetes mellitus [1].

Previously, the knowledge about the complications of diabetes was based mainly on the morphological changes and clinical presentation. However, a clearer and more precise understanding of these complications have stemmed from some extensive research work in the past decades.

The condition of skin has long provided clues to the presence of diabetes. This includes frequent infections, dryness, nonspecific pruritus etc. In the last few years many new associations between diabetes mellitus and the skin have been noted and we have a better understanding now of the pathophysiology of some diabetic complications. Microcirculatory alteration, glycosylation of different proteins with production of advanced glycosylated end product and their subsequent deposition in the basement membrane and alterations in the lipid profile results in changes of the skin condition of diabetic patients [2]. Diabetes also alters endothelial functions [3]. Diabetes and/or hypercholesterolemia alter the skin structure as well as the function, and fish oil-supplementation scavenges the free radicals and changes the skin structure and function of rats subjected to diabetes and or hypercholesterolemia [4]. In animal studies, it was shown that maternal diabetes and/or hypercholesterolemia increased the average deformation of hair follicles, vacuolation, and degeneration of epidermal cell layers [5]. Ultra-structure and size of human corneocytes in the upper stratum corneum layer of skin are changed in diabetic subjects [6]. Though hyperglycemia is pivotal, the above factors independently and/or complimentarily along with hyperglycemia are being considered in the causal role in the morbidity of diabetic complications.

*Corresponding author: Dr. Arjun Baidya, Assistant Professor, Department of Endocrinology, N.R.S Medical College, AJC Bose Road, Kolkata-700020, Tel: +919433154618; E-mail: arjun.baidya@gmail.com

Received February 11, 2018; Accepted March 21, 2018; Published March 26 2018

Citation: Baidya A, Halder AK, Datta PK (2018) Spectrum of Cutaneous Manifestations of Diabetes Mellitus: An Observational Study from a Tertiary Care Hospital. J Diabetes Metab 9: 791. doi: 10.4172/2155-6156.1000791

Copyright: @ 2018 Baidya A, et al. This is an open-access article distributed under the terms of the Creative Commons Attribution License, which permits unrestricted use, distribution, and reproduction in any medium, provided the original author and source are credited. 
A significant number of diabetic patients in skin out door and diabetic clinics are presenting different types of dermatological manifestations. This study was conducted to learn about demographic characteristics of subjects with diabetes having cutaneous manifestations and to note the clinical patterns of dermatological manifestations of DM.

\section{Materials and Methods}

This observational study was done in the outpatient department of dermatology, Medical College \& Hospital, Kolkata, India. Sixty consecutive subjects of either sex diagnosed with diabetes and having dermatological problems were recruited. Patients who had skin changes resulting from known causes or disease other than diabetes mellitus and subjects with acute complications of diabetes were excluded. Approval from the institutional ethics committee and informed consents from all individual participants or from their legal guardians in the case of minors were obtained.

History taking, general and systemic examination including examination of skin, oral and genital mucosa, hairs and nails were done. All subjects were then put on routine investigations and some special investigations like tissue fluid/pus for culture and Gram's stain, $10 \%$ potassium hydroxide $(\mathrm{KOH})$ preparation; skin or tissue biopsy for histopathological examination were done, if indicated. Descriptive statistics were presented in the form of mean, standard deviation and range for data on continuous scale depending on the distribution of data. Categorical measurements are presented in percent numbers (\%).

\section{Results}

In this study out of 60 subjects, 34 were male and 26 were female (9 type $1 \mathrm{DM}$ and 51 type $2 \mathrm{DM}$ ). Mean age in type $1 \mathrm{DM}$ was 22 years (range 7 to 34 years) and in type $2 \mathrm{DM}$ was 45 years (range 32 to 62 years), respectively. Mean duration of diabetes in type 1 and type 2 DM was 8 years $( \pm 3.6)$ and 7 years $( \pm 3.8)$, respectively (Table 1$)$. In this study, the cutaneous manifestation of diabetes mellitus has been discussed under four major groups (Table $2 \mathrm{a}$ and $2 \mathrm{~b}$ ). Various types of skin lesions according to type of DM observed are presented in Table 1 , $2 \mathrm{a}$ and $2 \mathrm{~b}$. Various types of skin infections observed are listed in Table 3.

\begin{tabular}{|l|c|c|c|c|}
\hline $\begin{array}{l}\text { Group of cutaneous } \\
\text { diseases }\end{array}$ & Type 1 DM & Type 2 DM & $\begin{array}{c}\text { Total } \\
\text { cases }\end{array}$ & $\%$ \\
\hline $\begin{array}{l}\text { Diseases due to gross metabolic } \\
\text { changes }\end{array}$ & 5 & 29 & 34 & $56.60 \%$ \\
\hline $\begin{array}{l}\text { Diseases due to chronic degenerative } \\
\text { changes }\end{array}$ & 2 & 20 & 22 & $36.60 \%$ \\
\hline $\begin{array}{l}\text { Diseases commonly associated with } \\
\text { DM }\end{array}$ & 3 & 23 & 26 & $43.30 \%$ \\
\hline $\begin{array}{l}\text { Disease/changes due to treatment } \\
\text { of DM }\end{array}$ & 2 & 5 & 7 & $11.60 \%$ \\
\hline
\end{tabular}

Table 1: Major groups of cutaneous diseases according to types of diabetes mellitus.

\begin{tabular}{|l|c|c|c|c|c|}
\hline Name of skin disease & Type 1 DM & Type 2 DM & Total & $\%$ \\
\hline Cutaneous diseases due to gross metabolic changes & \multicolumn{2}{|c|}{} \\
\hline Cutaneous infections & 5 & 25 & 30 & $50 \%$ \\
\hline Xanthelasma palpibrarum & 0 & 4 & 4 & $6.60 \%$ \\
\hline Xanthoma & 1 & 0 & 1 & $1.60 \%$ \\
\hline Cutaneous diseases due to chronic degenerative and vascular changes \\
\hline Diabetic dermopathy & 2 & 10 & 12 & $20 \%$ \\
\hline Diabetic foot ulcer and gangrene & 1 & 7 & 8 & $13.30 \%$ \\
\hline Miscellaneous diseases & 1 & 1 & 2 & $3.30 \%$ \\
\hline
\end{tabular}

Table 2a: The cutaneous manifestation of diabetes mellitus due to gross metabolic changes and chronic degenerative and vascular changes.

\begin{tabular}{|c|c|c|c|c|}
\hline Name of skin disease & Type 1 DM & Type 2 DM & Total & $\%$ \\
\hline \multicolumn{5}{|c|}{ Cutaneous diseases commonly associated with diabetes mellitus } \\
\hline $\begin{array}{l}\text { Pigmented purpuric dematosis } \\
\text { (PPD) }\end{array}$ & 0 & 4 & 4 & $6.60 \%$ \\
\hline Acanthosis nigricans (AN) & 0 & 4 & 4 & $6.60 \%$ \\
\hline Psoriasis (Ps) & 0 & 2 & 2 & $3.30 \%$ \\
\hline Vitiligo & 0 & 4 & 4 & $6.60 \%$ \\
\hline Lichen planus (LP) & 0 & 2 & 2 & $3.30 \%$ \\
\hline Skin tags & 0 & 6 & 6 & $10.00 \%$ \\
\hline Porokeratosis & 0 & 2 & 2 & $3.30 \%$ \\
\hline Pruritus and Xerosis & 3 & 5 & 8 & $13.30 \%$ \\
\hline $\begin{array}{l}\text { Sclerosis, thick skin and } \\
\text { contractures. }\end{array}$ & 1 & 3 & 4 & $6.60 \%$ \\
\hline \multicolumn{5}{|c|}{ Cutaneous manifestations due to treatment of diabetes mellitus } \\
\hline Lipodystrophy & 1 & 1 & 2 & $3 \%$ \\
\hline Skin rashes & 2 & 2 & 4 & $6.60 \%$ \\
\hline Scar with abscess & 0 & 1 & 1 & $1.60 \%$ \\
\hline
\end{tabular}

Table 2b: The cutaneous manifestation of diabetes mellitus associated with diabetes mellitus and its treatment.

\begin{tabular}{|l|c|c|c|c|}
\hline Type of infection & Type 1 DM & Type 2 DM & Total no. & $\%$ \\
\hline Pyogenic infections & 3 & 11 & 14 & $46.70 \%$ \\
\hline Candidiasis & 1 & 6 & 7 & $23.30 \%$ \\
\hline Dermatophytoses & 1 & 8 & 9 & $30 \%$ \\
\hline Total no. & 5 & 25 & 30 & $100 \%$ \\
\hline
\end{tabular}

Table 3: Frequency of different type of cutaneous infections.

\section{Discussion}

In our study, diseases attributed to gross metabolic changes including infections were the most frequently occurring skin diseases (56.6\%) followed by the diseases frequently associated with DM (43.3\%). Diseases due to degeneration with vascular changes and diseases due to treatment complications of DM were $36.6 \%$ and $11.6 \%$, respectively. Similar observation was seen in a study by Wahid et al. [7].

Incidence of cutaneous infection varies from one series to another. However, every investigator found chances of skin infection to be much more common in uncontrolled diabetics than the controlled one. In a study Paron et al., skin infections occurred in $20 \%$ to $50 \%$ cases of poorly controlled diabetes, most commonly in type 2 diabetics [8]. A similar study by Wahid et al. demonstrated cutaneous infections in $49 \%$ of all diabetic patients [7]. In this study, infective dermatological changes were found in $50 \%$ of all cases ( 5 type 1 and 25 type 2 ) and were mostly associated with poor glycemic control (Table 2a). Bacterial infections mainly of staphylococcal and streptococcal infections constituted $46.7 \%$ (14 cases) of all infections in both types of DM. Candidiasis in the form of intertrigo, paronychia, candidal vulvovaginitis and balanoposthitis constituted $23.3 \%$ of all infections. Different form of dermatophytosis (including toe nail onychomycosis) constituted $30 \%$ of all infections (Table 3). Most authors believe that mucocutaneous candida infections occur more frequently among patients with DM, especially those with poorly controlled disease as stated by Vazquez et al. [9] Stone et al. observed that $9.3 \%$ of diabetics showed candidiasis, compared to only $3.4 \%$ of nondiabetics [10]. However, the present study clearly showed the increased incidence (11.6\%) of different form of candidiasis and 23.3\% among the cutaneous infections, although two studies by LugoSomolinos et al. [11] and Romano et al. [12] failed to demonstrate an overall increased prevalence of dermatophyte infections in patients with DM compared to control subjects. A recent study by Gupta et al. [13] demonstrated the rate of dermatophyte infections, especially toe nail onychomycosis, to be 2.77 times greater for patients with DM as 
compared to controls. The present study exhibited an overall $15 \%$ prevalence of dermatophyte infections, which is further doubled among cutaneous infections (including four cases of toe nail onychomycosis).

Eruptive xanthomas - though rare - are found mainly in untreated $\mathrm{DM}$ and hypertriglyceridemia, whereas xanthelasma palpebrarum is very frequently associated with DM and hyperlipidaemia. Eruptive xanthoma mainly occurs in type $1 \mathrm{DM}$, but no definite relations between DM and hyperlipidaemia can be ascertained as they are also found in normal subjects [8]. This study showed one case of eruptive xanthoma in type $1 \mathrm{DM}$ and four cases (6.6\%) xanthelasma palpebrarum in type 2 DM (Table 2a).

Dermopathy, also called 'shin spot,' is said to be the most common cutaneous finding in diabetes. In 1982, Huntley concluded that it would be present in about $50 \%$ of known diabetic patients [14]. In a recent population based study from Sweden, diabetic dermopathy was found in $33 \%$ and $39 \%$ in patients with type $1 \mathrm{DM}$ and type $2 \mathrm{DM}$, respectively. On the contrary, the healthy control group showed only $2 \%$ of similar lesions on their shins stated by Bossen et al. [15]. In our study, shin spots were detected in 12 cases (20\%), much less than the incidence reported in different literature (Table 2a). Darker skin complexion may be one of the possible factors for the low incidence rate in our country.

It has been estimated that $15 \%$ of individuals with DM will develop lower extremity ulcers [16]. This study showed that total eight cases (13.3\%) of DM were with diabetic foot ulceration or gangrene and mostly under poorly controlled type $2 \mathrm{DM}$ (Table 2a).

Pigmented purpuric dermatosis (PPD) is an uncommon condition associated with diabetes but a definite relation between PPD and DM has not been proven. Approximately half of these patients had coexisting atrophic pigmented macules (diabetic dermopathy). The lower extremity purpura found in diabetic patients may resemble the purpura of Schamberg's disease as stated by Lithner [17]. In this study total four cases $(6.6 \%)$ of PPD were observed and mostly connected with type $2 \mathrm{DM}$ (Table $2 \mathrm{~b})$.

In a study of 223 patients with acanthosis nigricans, nearly $50 \%$ of patients in their fifth decade had type $2 \mathrm{DM}$, whereas only four of the 99 patients under 20 years had documented type 2 DM (Figure 1). Acanthosis nigricans may be considered as a prognostic indicator for the development of type $2 \mathrm{DM}$ as stated by Stuart CA et al. [18] Again acanthosis nigricans occurred in $66 \%$ of children who weighed $200 \%$ of their ideal body weight. This study also showed that total four cases (6.6\%) of acanthosis nigricans existed, all in type 2DM and associated with obesity (Table $2 b$ ).

In this study, incidence of psoriasis was 3.3\% lower than the rate (8.8\%) reported in Anand's study [19]. Incidence of vitiligo was 6.6\% (4 cases) and all cases were in type $2 \mathrm{DM}$ (Table $2 \mathrm{~b}$ ). A similar frequency of 4.5-7.7\% has been reported in type $2 \mathrm{DM}$ and mostly seen after the age of 40 years by Jelinek JE [20] and Sharma SC [21]

In the diabetic population, the incidence of lichen planus has been recorded as $1.6 \%$ by Lozada-Nur et al. [22] and Jain et al. [23]. This study showed that incidence of lichen planus was $3.3 \%$ (Table $2 \mathrm{~b}$ ).

Skin tags often associated with obesity were observed in $26 \%$ of type $2 \mathrm{DM}$ as stated by Kahana et al. [24]. This study showed that incidence of skin tags in diabetes was $10 \%$ (six cases) and mostly associated with obesity, acanthosis nigricans and type $2 \mathrm{DM}$ (Table $2 \mathrm{~b}$ and Figure 1).

Porokeratosis, especially superficial disseminated type, is more common in type $2 \mathrm{DM}$ as reported by Pavithran [25]. This study also showed two cases of porokeratosis of superficial disseminated form in type $2 \mathrm{DM}$ (Table $2 \mathrm{~b}$ )

Recent studies showed localized ano-genital pruritus to be more common in diabetes and pruritus and xerosis are coexisting in DM as stated by Neilly et al. [26]. In a study of 170 diabetic cases conducted by Anand [19], it was revealed that incidence of pruritus of unexplained origin was found in $40 \%$ of these diabetics. In this study pruritus of unexplained origin was $13.3 \%$ and much less than that of Anand's study (Table 2b) [19].

Diabetic bullae are very rare findings and approximately $0.5 \%$ of diabetic develop diabetic bullae or bullosis diabeticorum, a clinically distinct diabetic marker. These bullae have only been reported in adult (40 to 77 years old) and more commonly in long standing diabetes with neuropathy as stated by Paron et al. [8]. This study showed bulla in only one male patient of type $2 \mathrm{DM}$ with a prolonged duration (Table 2a).

Scleroderma-like skin changes and limited joint mobility are frequently found in diabetes of both types. The prevalence of this syndrome varies from $8 \%$ to $50 \%$ of diabetes as stated by Brik et al. [27].

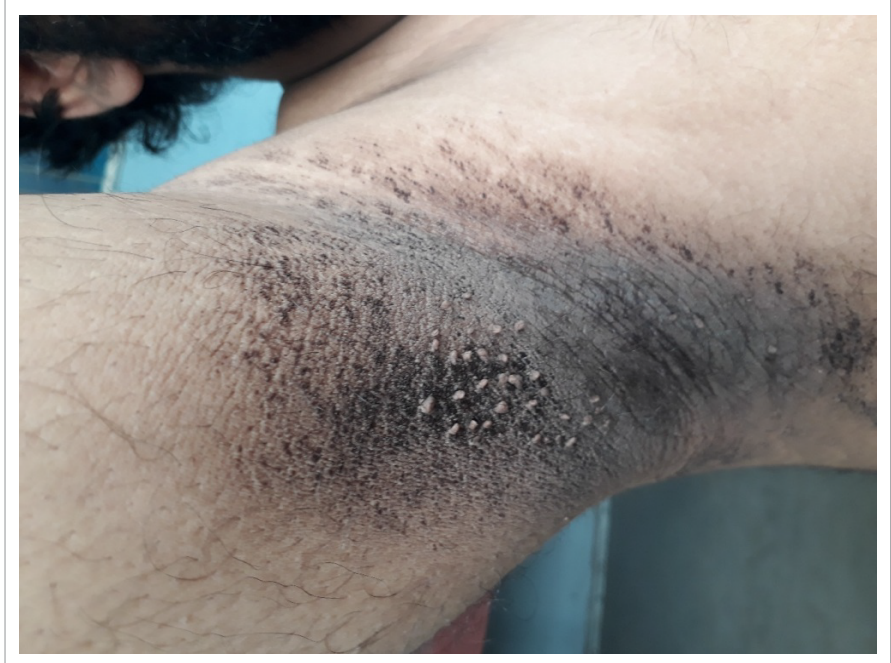

Figure 1: Showing acanthosis nigricans and skin tags.

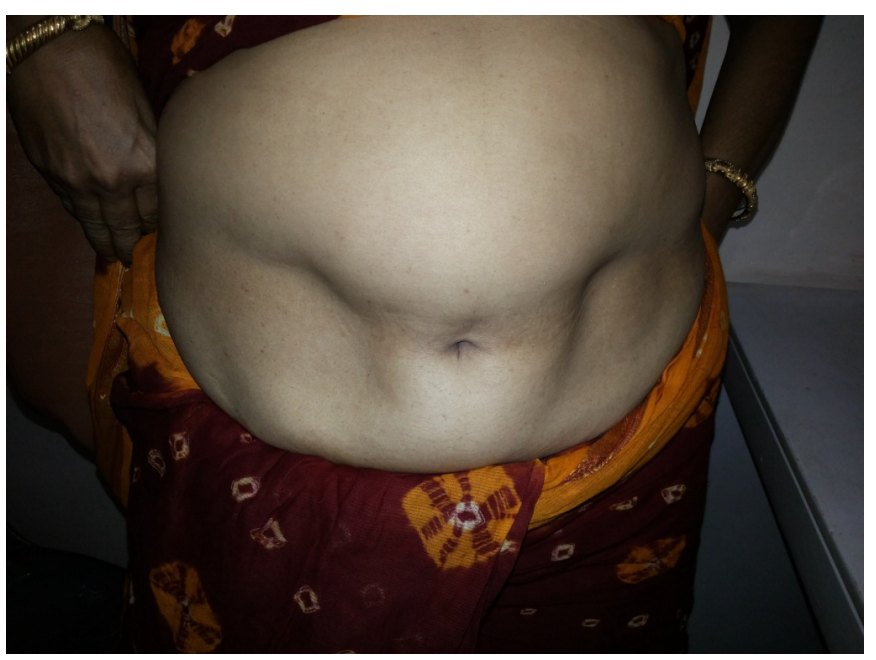

Figure 2: Showing Lipoatrophy. 
This study showed $6.6 \%$ (four cases) diabetic thick skin or scleroderma (Table 2b).

Lipodystrophy either in the form of lipoatrophy or lipohypertrophy is associated with insulin of older type and are rare with the newer, purified human insulin now a days. This study showed two cases of lipoatrophy in patients treated with human insulin (Table 2b and Figure 2).

The prevalence of skin rashes in patients on oral hypoglycemic agents (OHAs) are $1 \%$ to $5 \%$ mainly with sulfonylureas, and maculopapular rash is the commonest form as stated by Paron et al. [3]. This study showed four cases of skin rashes (6.6\%) with patients on OHAs (Table 2b).

\section{Conclusion}

The small sample size is a limitation of our study. However, this study has provided some insight into the pattern of cutaneous manifestations of diabetes mellitus, and its complications more frequently occurred in diabetes of poor glycemic control with longer duration and were associated with obesity and hyperlipidemia. A larger prospective study covering more patients, more parameters, more sophisticated gadgets will further consolidate our main objective that management of diabetes is a holistic one and that too should focus due attention on skin complications.

\section{Funding}

Nothing to declare.

\section{Statement of Authorship}

All authors have given approval to the final version submitted.

\section{Conflict of Interest}

Authors declare no conflict of interests.

\section{References}

1. Albin J, Rifkin H (1986) The diabetic state. In Jelinek JE edn: The skin in diabetes. (1stedn), Philadelphia.

2. Sibbald RG, Schachter RK (1984) The skin and diabetes mellitus. Int J Dermatol 23: 567-584.

3. Ciccone MM, Scicchitano P, Cameli M, Cecere A, Cortese F, et al. (2014) Endothelial Function in Pre-diabetes, Diabetes and Diabetic Cardiomyopathy: A Review. J Diabetes Metab 5: 364

4. El-Sayyad HIH, Abdraboh ME, Aljebali AMA (2015) Positive Impact of Fish Oil on Diabetic and Hypercholestrolemic Skin Disorders. J Nutri Health 1: 8.

5. El-Sayyad HI, Khalifa SA, Fouda YA, Yonis AS (2012) Effects of diabetes and or hypercholesterolemia on rat foetuses skin development. J Nutrition 28: 698-706.
6. Stücker M Licht M, Heise HM (2015) Surface Ultra-Structure and Size of Human Corneocytes from Upper Stratum Corneum Layers of Normal and Diabetic Subjects with Discussion of Cohesion Aspects. J Diabetes Metab 6: 603.

7. Wahid Z, Kanjee A (1989) Cutaneous manifestation of diabetes mellitus. The J Pak Med Assoc 48: 304-305.

8. Paron NG, Lambert PW (2000) Cutaneous manifestations of diabetes mellitus Prim Care 27: 371-383.

9. Vazquez JA, Sobel JD (1995) Fungal infections in diabetes. Infect Dis Clin North Am 9: 97-116.

10. Stone OJ, Mullins JF (1963) Incidence of chronic paronychia. JAMA 186: 71

11. Lugo-Somolinos A, Sanchez J (1992) Prevalence of dermatophytosis in patients with diabetes. J Am Acad Dermatol 26: 408-410.

12. Romano C, Massai L, Asta F, Signorini AM (2001) Prevalence of dermatophytic skin and nail infections in diabetic patients. Mycoses 44: 83.

13. Gupta AK, Konnikov N, Macdonala P, Rich P, Rodger NW, et al. (1998) Prevalence and epidemiology of toenail onychomycosis in diabetic subject: A multicentic survey. Br J Dermatol 139: 665

14. Huntley AC (1982) The cutaneous manifestations of diabetes mellitus. J Am Acad Dermatol 7: 427-455.

15. Bossen B (1990) The epidemiology of foot lesions in diabetic patients aged 15-50 years. Diabet Med 7: 438

16. Reiber GE (1996) The epidemiology of diabetic foot problem. Diabet Med 13: 56 .

17. Lithner F (1974) Cutaneous erythema with or without necrosis. Localised to the legs and feet-a lesion in elderly diabetics. Acta Med Scand 196: 333-342.

18. Stuart CA, Gilkison CR, Smith MM, Bosma AM, Keenan SB, et al. (1998) Acanthosis nigricans as a risk factor for noninsulin dependent diabetes mellitus. Clin Pediatr 37: 73-80.

19. Anand LC (1978) Assessment of diabetic state in various skin disorders usually associated with hyperglycemia. Indian J Dermatol veneroeol Leprol 44: 95-102.

20. Jelinek JE (1994) Cutaneous manifestations of diabetes mellitus. Int Dermatol 33: 605-617.

21. Sharma SC (1991) Autoimmune and cutaneous association of various types of vitiligo. Indian J Dermatol Venereol Leprol 57: 107-108.

22. Lozada-Nur F, Miranda C (1997) Oral lichen planus: epidemiology, Clinica characteristics, and associated diseases. Semin Cutan Med Surg 16: 273-277.

23. Jain RK, Datta P, Sing R (1983) Lichen planus and glucose tolerance. Indian J Dermatol venereol Leprol 49: 147-149.

24. Kahana M, Grossman E, Feintein A (1987) Skin tags: A cutaneous marker for diabetes mellitus. Acta Derm venerol 67: 175-177.

25. Pavithran K (1991) Superficial disseminated porokeratosis with diabetes mellitus. Indian J Dermatol Venereol Leprol 57: 36-37.

26. Neilly JB, Martin A, Simpson N, MacCuish AC (1986) Pruritus in diabetes mellitus: investigation of prevalence and co-relation with diabetes control. Diabetes Care 9: 273-275.

27. Brik R, Berant M, Vardi $P$ (1991) The scleroderma like syndrome of insulin dependent diabetes mellitus. Diabetes Metab Rev 7: 120-128. 